\title{
Nonlinear Stability of Rarefaction Waves for Compressible Navier Stokes Equations
}

Tai-Ping Liu $^{1 \star}$ and Zhouping $\mathrm{Xin}^{2 \star \star}$

${ }^{1}$ Department of Mathematics, University of Maryland, College Park, MD 20742, USA

${ }^{2}$ Department of Mathematics, University of Michigan, Ann Arbor, MI 48109, USA

\begin{abstract}
It is shown that expansion waves for the compressible Navier-Stokes equations are nonlinearly stable. The expansion waves are constructed for the compressible Euler equations based on the inviscid Burgers equation. Our result shows that Navier-Stokes equations and Euler equations are timeasymptotically equivalent on the level of expansion waves. The result is proved using the energy method, making essential use of the expansion of the underlining nonlinear waves and the specific form of the constitutive eqution for a polytropic gas.
\end{abstract}

\section{Introduction}

Consider one-dimensional compressible Navier-Stokes equations in the Lagrangian Coordinates,

$$
\begin{aligned}
v_{t}-u_{x} & =0, \\
u_{t}+p_{x} & =\left(\mu u_{x}(v)\right)_{x}, \\
\left(e+\frac{u^{2}}{2}\right)_{t}+(p u)_{x} & =\left(\kappa \theta_{x} / v+\mu u u_{x} / v\right)_{x},
\end{aligned}
$$

where $v, u, p, e$ and $\theta$ are, respectively, the specific volume, velocity, pressure, internal energy and the temperatures of the gas, and the positive constants $\mu, \kappa$ are the viscosity and heat conductivity coefficients. The gas is assumed to be polytropic:

$$
p=R \theta / v=A \exp \left(s / c_{\vartheta}\right) v^{-\gamma}, \quad e=\frac{R \theta}{\gamma-1},
$$

where $s$ is the entropy, $R>0$ the gas constant, $\gamma>1$ the adiabatic constant, $c_{v}=R /(\gamma-1)$ the specific heat, and $A$ a positive constant. We are interested in the

\footnotetext{
* Supported in part by NSF Grant DMS-87-03971 and Army Grant DAAL03-87-K-0063

$\star \star$ Supported in part by Army Grant DAAL03-87-K-0063
} 
expansion waves. For this, it is convenient to work with the equation for the entropy

$$
s_{t}=\frac{1}{p v} \frac{\kappa}{c_{v}}\left(p s_{x}\right)_{x}-\frac{\kappa(\gamma-1)}{p v}\left(\frac{p v_{x}}{v}\right)_{x}+\frac{R \mu u_{x}^{2}}{p v^{2}}
$$

Equations $(1.1)_{1},(1.1)_{2},(1.1)_{3}$ and $(1.1)_{1},(1.1)_{2},(1.1)_{3}^{\prime}$ are equivalent.

Our purpose is to show that expansion waves for (1.1) are nonlinearly stable. For expansion waves the right-hand side of (1.1) decays faster than each term in the left-hand side. Therefore the compressible Navier-Stokes Eq. (1.1) may be replaced, time-asymptotically for expansion waves, by the compressible Euler equations

$$
\begin{aligned}
v_{t}-u_{x} & =0, \\
u_{t}+p_{x} & =0, \\
s_{t} & =0 .
\end{aligned}
$$

There are two families of expansion (rarefaction) waves for (1.3), CourantFriedrichs [1]. We will describe only the 1-rarefaction waves, which are characterized by

$$
\begin{aligned}
& s \text { and } u+\frac{2}{\gamma-1}(\gamma v p)^{1 / 2} \text { constant in }(x, t), \text { and } \\
& \lambda_{1}(v, s)(x, t) \equiv-\left(\gamma p v^{-1}\right)^{1 / 2} \text { increasing in } x .
\end{aligned}
$$

Suppose that the end states of the initial data for (1.3) satisfy

$$
\begin{aligned}
& s_{ \pm} \equiv s( \pm \infty, 0)=s_{0}, \\
& u_{ \pm}+\frac{2}{\gamma-1}\left(\gamma v_{ \pm} p_{ \pm}\right)^{1 / 2} \equiv\left(u+\frac{2}{\gamma-1}(\gamma v p)^{1 / 2}\right)( \pm \infty, 0)=A_{0}, \\
& \lambda_{+} \equiv \lambda_{1}(v, s)(+\infty, 0)>\lambda_{1}(v, s)(-\infty, 0) \equiv \lambda_{-} .
\end{aligned}
$$

Rarefaction waves for (1.3) with same end states converge to each other. For definiteness, we choose a particular 1-rarefaction wave of $(1.3)(u, v, s)(x, t)=$ $(\bar{u}, \bar{v}, \bar{s})(x, t):$ with initial value

$$
\begin{aligned}
& \bar{s}(x, 0)=0, \\
& \bar{u}+\frac{2}{\gamma-1}(\gamma \bar{v} \bar{p})^{1 / 2}(x, 0)=A_{0},(\bar{u}, \bar{v})( \pm \infty, 0)=\left(u_{ \pm}, v_{ \pm}\right), v_{+}>0, v_{-}>0, \\
& \lambda_{1}(\bar{v}, 0)(x, 0)=\left(\lambda_{+}+\lambda_{-}\right) / 2+\left(\left(\lambda_{+}-\lambda_{-}\right) / 2\right) \tanh x,
\end{aligned}
$$

where, for simplicity, we have set $s_{0}=0$. Our main result is the following stability theorem.

Theorem 1. Suppose that $(\bar{u}, \bar{v}, 0)$ is the rarefaction wave of the compressible Euler equation (1.3), (1.4) and that the initial data $(u, v, s)(x, 0)=\left(u_{0}, v_{0}, s_{0}\right)(x)$ of the compressible Navier-Stokes equations (1.1) satisfy $\left(u_{0}, v_{0}, s_{0}\right)( \pm \infty)=\left(u_{ \pm}, v_{ \pm}, 0\right)$ and that at $t=0$,

$$
\begin{aligned}
\delta \equiv & \left|v_{+}-v_{-}\right|+\left|u_{+}-u_{-}\right|+\left\|v_{0}-\bar{u}\right\|_{L_{2}}^{2}+\left\|u_{0}-\bar{u}\right\|_{L_{2}}^{2}+\left\|s_{0}\right\|_{L_{2}}^{2} \\
& +\left\|\left(v_{0 x}, u_{0 x}, s_{0 x}\right)\right\|_{L_{2}}^{2}
\end{aligned}
$$


is sufficiently small. Then the solution $(u, v, s)(x, t)$ of $(1.1)$ exists for all time and

$$
\left.\lim _{t \rightarrow \infty} \sup _{-\infty<x<\infty}\{\mid v-\vec{v})(x, t)|+|(u-\vec{u})(x, t)|+| s(x, t) \mid\right\}=0 .
$$

Analogous results also hold for linear superposition of 1-rarefaction and 3-rarefaction waves by combining our technique here and the characteristic-energy method of Liu [3]. The stability of expansion waves for the isentropic flow has been proved by Matzumura and Nishihara [5]; see also [4] and [6] for stability of expansion waves for other systems. Expansion waves and compression waves for (1.1) are both stable, but in a markedly different sense. Expansion waves are stable in the $\sup \left(L_{\infty}\right)$ norm but not in the integral $\left(L_{1}\right)$ norm, while compression waves are supposed to be stable in both norms, cf. [3]. As a consequence the stability analysis for expansion waves differs in some basic way from that for compression waves. Although we use the energy method as in previous works $[5,4,6]$, we do not use the smallness of $\delta,(1.5)$, to control some of the first order terms. Instead, we used the entropy equation $(1.3)^{\prime}$ and the specific form of the constitutive relation (1.2) for the final, and crucial, energy estimate; see the proof of Proposition 3.5 .

\section{Expansion Waves}

The characteristics for the Euler equations (1.3) are

$$
\lambda_{1}=-\left(\gamma p v^{-1}\right)^{1 / 2}, \quad \lambda_{2}=0, \quad \lambda_{3}=\left(\gamma p v^{-1}\right)^{1 / 2} .
$$

It can be shown easily that because the 1-rarefaction waves form (1.3) take value along the 1-characteristic direction, the characteristic speed $\lambda_{1}$ satisfies the inviscid Burgers equation, [2]:

$$
\frac{\partial \lambda_{1}}{\partial t}+\lambda_{1} \frac{\partial \lambda_{1}}{\partial x}=0
$$

The specific choice of the initial data (1.4) yields, by characteristic method for (2.1),

$$
\begin{aligned}
\lambda_{1}(x, t)= & -\frac{1}{2}\left(\left(\gamma p_{-} v_{-}{ }^{-1}\right)^{1 / 2}-\left(\gamma p_{+} v_{+}{ }^{-1}\right)^{1 / 2}\right) \tanh \left(x-\lambda_{1}(x, t) t\right) \\
& -\frac{1}{2}\left(\left(\gamma p_{+} v_{+}{ }^{-1}\right)^{1 / 2}+\left(\gamma p_{-} v_{-}{ }^{-1}\right)^{1 / 2}\right) \\
= & -\frac{1}{2}\left(\left(A \gamma v_{-}{ }^{-\gamma-1}\right)^{1 / 2}-\left(A \gamma v_{+}{ }^{-\gamma-1}\right)^{1 / 2}\right) \tanh \left(x-\lambda_{1}(x, t) t\right) \\
& -\frac{1}{2}\left(\left(A \gamma v_{+}{ }^{-\gamma-1}\right)^{1 / 2}+\left(A \gamma v_{-}{ }^{-\gamma-1}\right)^{1 / 2}\right) .
\end{aligned}
$$

This can be solved for $\lambda_{1}(x, t)$. $\lambda_{1}$ rarefies in that $\partial \lambda_{1} / \partial x$ decays at the rate $t^{-1}$. The other physical quantities are obtained from

$$
\begin{aligned}
\bar{s}(x, t) & =0, \\
\bar{u}+\frac{2}{\gamma-1}(\gamma \bar{v} \bar{p})^{1 / 2}(x, t) & =A_{0}, \\
-\left(\gamma \bar{v} \bar{p}^{-1}\right)^{1 / 2}(x, t) & =\lambda_{1}(x, t) .
\end{aligned}
$$

Thus $(\bar{u}, \bar{v})$ also rarefies. It follows from these identities that their $L_{p}$ norms decay algebraically. 
Lemma 2.1. Let $\delta_{0}=\left|v_{+}-v_{-}\right|+\left|u_{+}-u_{-}\right|$, then for bounds $0(1)$ independent of $t$

(i) $\tilde{v}_{t}(x, t)>0, v_{-}>v_{+}>0$,

(ii) $\left\|(\bar{v}, \bar{u})_{x}\right\|_{L_{p}(x)}=O(1) \delta_{0}^{1 / p}(1+t)^{-1+1 / p}\left\|\bar{u}_{x}\right\|_{L_{\infty}(x)}=O(1) \delta_{0}, 1 \leqq p \leqq \infty$,

(iii) $\left\|\frac{\partial^{l}}{\partial x^{l}}(\bar{v}, \bar{u})\right\|_{L_{p}(x)}=O(1) \min \left(\delta_{0},(1+t)^{-1}\right), 1 \leqq p<\infty, l \geqq 2$,

(iv) $\left\|(\bar{v}, \bar{u})_{t}\right\|_{L_{\infty}(x)}=O(1)\|(\bar{v}, \bar{u})\|_{L^{\infty}(x)}$.

For the details of the proof, see Matzumura-Nishihara [5] and Xin [6].

\section{Energy Estimates}

In what follows $\|\cdot\|_{l}, l=0,1,2, \ldots$, denote the usual Sobolev norms for $H^{l}(x)$, $\|\cdot\| \equiv\|\cdot\|_{0},|\cdot| \equiv\|\cdot\|_{L_{\infty}(x)}$ and \|\|$_{L_{p}}$ the $L_{p}(x)$ norm, $1 \leqq p \leqq \infty$. Let $(u, v, s)(x, t)$ be the solution of (1.1) with initial value

$$
\begin{gathered}
(u, v, s)(x, 0)=\left(u_{0}, v_{0}, s_{0}\right)(x), \\
\left(u_{0}, v_{0}, s_{0}\right)( \pm \infty)=\left(u_{ \pm}, v_{ \pm}, 0\right),
\end{gathered}
$$

and $(\bar{u}, \bar{v}, \bar{s})(x, t)$ the solution (2.2) of (1.3) and (1.4). Set

$$
\phi \equiv v-\bar{v}, \quad \psi \equiv u-\bar{u} .
$$

Since $(u, v, s)$ satisfies $(1.1),(1.1)_{2}$ and $(1.1)_{3}^{\prime}$ and $(\bar{u}, \bar{v}, \bar{s})$ satisfies $(1.3)$ we have

$$
\begin{aligned}
& \phi_{t}-\psi_{x}=0 \text {, } \\
& \psi_{t}+\left(p(\varphi+\bar{v}, s)-p(\bar{v}, 0)_{x}\right)=\mu\left(\frac{\bar{u}_{x}+\psi_{x}}{\bar{v}+\varphi}\right)_{x}, \\
& s_{t}=\frac{1}{p(\varphi+\bar{v}, s)(\phi+\bar{v})} \frac{\kappa}{c_{v}}\left(p(\varphi+\bar{v}, s) s_{x}\right)_{x}+\frac{R \mu\left(\bar{u}_{x}+\psi_{x}\right)^{2}}{p(\varphi+\bar{v}, s)(\varphi+\bar{v})^{2}} \\
& -\frac{(\gamma-1) \kappa}{p(\varphi+\bar{v}, s)(\varphi+\bar{v})}\left(\frac{p(\varphi+\bar{v}, s)\left(\phi_{x}+\bar{v}_{x}\right)}{\varphi+\bar{v}}\right)_{x}, \\
& (\varphi, \psi, s)(x, 0) \equiv\left(\varphi_{0}, \psi_{0}, s_{0}\right)(x)=\left(v_{0}-\bar{v}, u_{0}-\bar{u}, s_{0}\right)(x) .
\end{aligned}
$$

From (1.5) we have

$$
\delta \equiv\left|v_{+}-v_{-}\right|+\left|u_{+}-u_{-}\right|+\left\|\left(\varphi_{0}, \psi_{0}, s_{0}\right)\right\|_{2} \ll 1 .
$$

For brevity we denote by

$$
\begin{aligned}
\bar{p} & \equiv p(\bar{v}, \bar{s})=p(\bar{v}, 0)=A \bar{v}^{-\gamma} \\
p & \equiv p(\varphi+\bar{v}, s)=A(\varphi+\bar{v})^{-\gamma} \exp \left(s / c_{v}\right), \\
N(t) & \equiv \sup _{0 \leqq t \leqq t}\|(\varphi, \psi, s)(\cdot, \tau)\|_{2} .
\end{aligned}
$$

Lemma 3.1. Suppose that $N(t)$ is small. Then

$$
\delta(t) \equiv \sup _{0 \leqq t \leqq t}\left|\left(\varphi, \psi, s, \psi_{x}, \psi_{x}, s_{x}\right)(\cdot, \tau)\right|=O(1) N(t)
$$


is small and $p(x, t)>p_{0}, \bar{v}+\varphi(x, t)>v_{0}$ for some $p_{0}>0, v_{0}>0$ and $-\infty<x<\infty$, $0 \leqq \tau \leqq t$.

The proof of the above lemma following easily from the Sobolev inequality, and that $p_{-}>0$ and $v_{-}>0$. In the estimates below $0(1)$ denotes functions which are uniformly bounded for all time. We start with the estimate for the entropy $s$ in the next lemma followed by the estimate for $\varphi, \psi$ in the following two lemmas. These estimates are then put together in Proposition 3.5, whose proof contains the key new ingredient in our theory. In the following lemma we always assumed that for some $\varepsilon$, sufficiently small,

$$
N(t) \leqq \varepsilon .
$$

\section{Lemma 3.2.}

$$
\begin{aligned}
& \frac{1}{2} \int_{-\infty}^{\infty} s^{2}(t) d x+\frac{\kappa(\gamma-1)}{R v_{-}} \int_{0}^{t} \int_{-\infty}^{\infty} s_{x}^{2} d x d t-\frac{\kappa(\gamma-1)}{v_{-}^{2}} \int_{0}^{t} \int_{-\infty}^{\infty} s_{x} \varphi_{x} d x d t \\
& \quad=0(1)\left(\delta_{0}+N^{1 / 2}(t)\right)\left(\int_{0}^{t} \int_{-\infty}^{\infty}\left(\phi_{x}^{2}+\psi_{x}^{2}+s_{x}^{2}\right) d x d t+\delta_{0}^{1 / 6}\right)+\frac{1}{2} \int_{-\infty}^{\infty} s^{2}(0) d t, \\
& s(t) \equiv s(x, t) \text { etc. }
\end{aligned}
$$

Proof. Multiply $(3.3)_{3}$ by $s(t)$ and integrate over $\mathbb{R}^{1} \times[0, t]$ to obtain

$$
\begin{aligned}
\frac{1}{2}\|s(t)\|^{2}-\frac{1}{2}\|s(0)\|^{2}+\int_{0}^{t} \int_{-\infty}^{\infty} \frac{\kappa}{c_{v}(\phi+\bar{v})} s_{x}^{2} d x d t \\
=\int_{0}^{t} \int_{-\infty}^{\infty}\left(\frac{-\kappa}{c_{v}}\left(\frac{1}{p(\phi+\bar{v})}\right)_{x} p s_{x} s+\frac{R \mu\left(\bar{u}_{x}+\psi_{x}\right)^{2}}{p(\phi+\bar{v})^{2}} s\right. \\
\left.\quad-\frac{(\gamma-1) \kappa}{p(\varphi+\bar{v}, s)}\left(\frac{p\left(\varphi_{x}+\bar{v}_{x}\right)}{\varphi+\bar{v}}\right)_{x} s\right) d x d t .
\end{aligned}
$$

We first estimate the second term on the right-hand side:

$$
\begin{aligned}
& R \mu \int_{0}^{t} \int_{-\infty}^{\infty} \frac{\left(\bar{u}_{x}+\psi_{x}\right)^{2}}{p(\phi+\bar{v})^{2}} s d x d t \leqq O(1) \iint|s|\left(\left|\bar{u}_{x}\right|^{2}+\left|\psi_{x}\right|^{2}\right) d x d t \\
& \quad \leqq O(1) \int_{0}^{t}\|s\|^{1 / 2}\left\|s_{x}\right\|^{1 / 2}\left\|\bar{u}_{x}\right\|^{2} d x d t+O(1) N(t) \int_{0}^{t}\left\|\psi_{x}\right\|^{2} d t \\
& \quad \leqq O(1) \int_{0}^{t}\|s\|^{1 / 2}\left(\frac{1}{4}\left\|s_{x}\right\|^{2}+\frac{3}{4}\left\|\bar{u}_{x}\right\|^{8 / 3}\right) d x d t+O(1) N(t) \int_{0}^{t}\left\|\psi_{x}\right\|^{2} d t \\
& \quad \leqq O(1) N^{1 / 2}(t) \int_{0}^{t} \int_{-\infty}^{\infty}\left(s_{x}^{2}+\psi_{x}^{2}\right) d x d t+O(1) N^{1 / 2}(t) \delta_{0}^{4 / 3}
\end{aligned}
$$

where we have used Lemma 3.1, Sobolev inequality, (3.6), Young's inequality, and (ii) of Lemma 2.1. Similarly the other two terms on the right-hand side of (3.8) are estimated as follows:

$\frac{\kappa}{c_{v}} \int_{0}^{t} \int_{-\infty}^{\infty}\left(\frac{1}{p(\phi+\bar{v})}\right) p s_{x} s d x d t$ 


$$
\begin{aligned}
& =\frac{-\kappa}{c_{v}} \int_{0}^{t} \int_{-\infty}^{\infty} \frac{s s_{x P}}{p(\phi+\bar{v})^{2}}\left(\left(p_{v}(v+\varphi)+p\right)\left(\bar{v}_{x}+\varphi_{x}\right)+p_{s}(\bar{v}+\varphi) s_{x}\right) d x d t \\
& \leqq O(1) \int_{0}^{t} \int_{-\infty}^{\infty}|s|\left(\bar{v}_{x}^{2}+s_{x}^{2}+\varphi_{x}^{2}\right) d x d t \\
& \leqq O(1) N^{1 / 2}(t) \int_{0}^{t} \int_{-\infty}^{\infty}\left(s_{x}^{2}+\varphi_{x}^{2}\right) d x d t+O(1) N^{1 / 2}(t) \delta_{0}^{3 / 4}, \\
& -\int_{0}^{t} \int_{-\infty}^{\infty} \frac{(\gamma-1) \kappa}{p(\varphi+\bar{v})}\left(\frac{p\left(\varphi_{x}+\bar{v}_{x}\right)}{\varphi+\bar{v}}\right)_{x} s d x d t \\
& =\int_{0}^{t} \int_{-\infty}^{\infty}\left(-\frac{(\gamma-1) \kappa}{p(\varphi+\bar{v})} s\left(\frac{p \bar{v}_{x}}{\varphi+\bar{v}}\right)_{x}+\frac{(\gamma-1) \kappa}{p(\varphi+\bar{v})^{2}} s_{x} \varphi_{x}-\frac{(\gamma-1) \kappa s p \phi_{x}(\phi(p+\bar{v}))_{x}}{(\varphi+\bar{v})^{3} p^{2}}\right) d x d t \\
& \leqq(\gamma-1) x \int_{0}^{t} \int_{-\infty}^{\infty}\left(\left(\frac{\left|s \bar{v}_{x x}\right|}{(\varphi+\bar{v})^{2}}\right)+\left|\frac{s}{p(\varphi+\bar{v})}\left(\frac{p}{\varphi+\bar{v}}\right)_{x} \bar{v}_{x}\right|+\frac{s_{x} \varphi_{x}}{v_{-}^{2}}\right) d x d t \\
& +\int_{0}^{t} \int_{-\infty}^{\infty}\left(O(1)\left|s_{x} \varphi_{x}\right|\left(|\varphi|+\delta_{0}\right)+O(1)\left|s \varphi_{x}\right|\left(\left|\phi_{x}\right|+\left|\bar{v}_{x}\right|+\left|s_{x}\right|\right)\right) d x d t \\
& \leqq(\gamma-1) \kappa \int_{0}^{t} \int_{-\infty}^{\infty}\left(O(1)\left|s \bar{v}_{x x}\right|+\frac{s_{x} \varphi_{x}}{v_{-}^{2}}+O(1)|s|\left(\varphi_{x}^{2}+\bar{v}_{x}^{2}+s_{x}^{2}\right)\right. \\
& \left.+O(1)\left(\delta_{0}+N(t)\right)\left(s_{x}^{2}+\varphi_{x}^{2}\right)\right) d x d t \\
& \leqslant(\gamma-1) \kappa \int_{0}^{t} \int_{-\infty}^{\infty} \frac{s_{x} \varphi_{x}}{v_{-}^{2}} d x d t+O(1) \int_{0}^{t} \int_{-\infty}^{\infty}\left|s \bar{v}_{x x}\right| d x d t \\
& +O(1)\left(\delta_{0}+N^{1 / 2}(t)\right) \int_{0}^{t} \int_{-\infty}^{\infty}\left(s_{x}^{2}+\varphi_{x}^{2}\right)+O(1) N^{1 / 2}(t) \delta_{0}^{4 / 3} .
\end{aligned}
$$

Next, from Sobolev, Young inequalities and (iii) of Lemma 2.1,

$$
\begin{aligned}
\int_{0}^{t} \int_{-\infty}^{\infty}\left|s \bar{v}_{x x}\right| d x d t & \leqq \int_{0}^{t}\|s\|^{1 / 2}\left\|s_{x}\right\|^{1 / 2}\left\|\bar{v}_{x x}\right\|_{L_{1}} d t \\
& \leqq \int_{0}^{t}\|s\|^{1 / 2}\left(\frac{1}{4}\left\|s_{x}\right\|^{2}+\frac{3}{4}\left\|\bar{v}_{x x}\right\|_{L_{1}}^{4 / 3}\right) d t \\
& \leqq O(1) N^{1 / 2}(t) \int_{0}^{t} \int_{-\infty}^{\infty} s_{x}^{2} d x d t+O(1) N^{1 / 2}(t) \delta_{0}^{1 / 6}
\end{aligned}
$$

The lemma follows from these estimate and (3.9).

Q.E.D.

\section{Lemma 3.3.}

$$
\begin{aligned}
& \int_{-\infty}^{\infty}\left(\frac{1}{2}|\psi(t)|^{2}+\frac{1}{2}\left|p_{v}^{-}\right||\phi(t)|^{2}-p_{s}^{-} s(t) \varphi(t)\right) d x \\
& \quad+\int_{0}^{t} \int_{-\infty}^{\infty}\left(\frac{\mu}{v} \psi_{x}^{2}+\frac{(\gamma-1) x_{x}}{v_{-}^{2}} p_{s}^{-} \varphi_{x}^{2}+\frac{1}{4}\left|p_{v v}^{-} \bar{v}_{t}\right| \varphi^{2}-\frac{(\gamma-1) \varkappa}{R v_{-}} p_{s}^{-} s_{x} \varphi_{x}\right) d x d t
\end{aligned}
$$




$$
\begin{aligned}
\leqq & O(1)\left\{\|\varphi(0)\|^{2}+\|\psi(0)\|^{2}+\|s(0)\|^{2}\right) \\
& +O(1)\left(\delta_{0}+N^{1 / 2}(t)(\| \varphi(t))\left\|^{2}+\right\| s(t) \|^{2}\right. \\
& +\int_{0}^{t} \int_{-\infty}^{\infty}\left(\left|\varphi_{x}\right|^{2}+\left|\psi_{x}\right|^{2}+\left|s_{x}\right|^{2}+\bar{v}_{t} \varphi^{2}\right) d x d t+N^{1 / 2}(t) \delta_{0}^{1 / 6} \\
p_{s}^{-} \equiv & p_{s}\left(v_{-}, 0\right), \quad \mathrm{p}_{v}^{-} \equiv p_{v}\left(v_{-}, 0\right), \quad p_{v v}^{-} \equiv p_{v v}\left(v_{-}, 0\right)
\end{aligned}
$$

Proof. Multiply $(3.3)_{1}$ by $p(\bar{v}+\varphi, s)$ and (3.32) by $\psi$ and integrate their sum to get

$$
\begin{gathered}
\frac{1}{2} \int_{-\infty}^{\infty}|\psi(t)|^{2} d x+\int_{0}^{t} \int_{-\infty}^{\infty}(p(\bar{v}, s)-p(\bar{v}+\varphi, s)) \varphi_{t}+\left(p(\bar{v}, 0)-p(\bar{v}, s) \varphi_{t} d x d t\right. \\
=\frac{1}{2} \int^{\infty}|\psi(0)|^{2} d x+\int_{0}^{t} \int_{-\infty}^{\infty}\left(\mu \psi\left(\frac{\psi_{x}}{\bar{v}+\varphi}\right)_{x}+\mu \psi\left(\frac{\bar{u}_{x}}{\bar{v}+\varphi}\right)_{x}\right) d x d t .
\end{gathered}
$$

We first estimate the right-hand side of (3.11) by parts:

$$
\begin{aligned}
\int_{0}^{t} \int_{-\infty}^{\infty} \mu \psi\left(\frac{\psi_{x}}{\bar{v}+\varphi}\right)_{x} & =-\int_{0}^{t} \int_{-\infty}^{\infty} \frac{\mu}{\bar{v}+\varphi} \psi_{x}^{2} d x d t \\
& =-\int_{0}^{t} \int_{-\infty}^{\infty}\left(\frac{\mu}{\bar{v}}+O(1)\left(\delta_{0}+N(t)\right)\right) \psi_{x}^{2} d x d t,
\end{aligned}
$$

and by Lemma 2.1, Sobolev and Young's inequalities:

$$
\begin{aligned}
& \int_{0}^{t} \int_{-\infty}^{\infty} \mu \psi\left(\frac{\bar{u}_{x}}{\bar{v}+\varphi}\right) d x d t \\
& \quad=\int_{0}^{t} \int_{-\infty}^{\infty} O(1)\left(\left|\psi \bar{u}_{x x}\right|+\left|\psi \bar{v}_{x}^{2}\right|+\left|\psi \bar{u}_{x} \varphi_{x}\right| d x d t\right. \\
& \quad=\int_{0}^{t} O(1)\left(\|\psi\|^{1 / 2}\left\|\psi_{x}\right\|^{1 / 2}\left(\left\|\bar{u}_{x x}\right\|+\left\|\bar{v}_{x}\right\|^{2}+\left\|\bar{u}_{x}\right\|\left\|\varphi_{x}\right\|\right) d t\right. \\
& \quad=O(1) N^{1 / 2}(t) \int_{0}^{t}\left(\left\|\psi_{x}\right\|^{2}+\left\|\bar{u}_{x x}\right\|^{4 / 3}+\left\|\bar{v}_{x}\right\|^{8 / 3}+\left\|\varphi_{x}\right\|^{2}+\left\|\bar{u}_{x}\right\|^{3}\right) d t \\
& \quad=O(1) N^{1 / 2}(t) \int_{0}^{t} \int_{-\infty}^{\infty}\left(\psi_{x}^{2}+\varphi_{x}^{2}\right) d x d t+O(1) N^{1 / 2}(t) \delta_{0}^{1 / 6} .
\end{aligned}
$$

Next we estimate the left-hand side of $(3.11)$. Since $p(v, s)$ is a smooth function we have from Taylor expansion that

$$
\begin{aligned}
& p(\bar{v}+\varphi, s)-p(\bar{v}, s)=p_{v}(\bar{v}, s) \varphi+Q_{1} \varphi^{2}, \\
& p(\bar{v}, s)-p(\bar{v}, 0)=p_{s}(\bar{v}, 0) s+Q_{2} s^{2}
\end{aligned}
$$

for some smooth functions $Q_{1} \equiv Q_{1}(\bar{v}, \varphi, s)$ and $Q_{2}=Q_{2}(\bar{v}, s)$. Using integration by parts, (i) of Lemma 2.1 and that $p_{v v}>0,(1.2)$, we have

$$
\begin{gathered}
\int_{0}^{t} \int_{-\infty}^{\infty} p[(\bar{v}, s)-p(\bar{v}+\varphi, s)] \varphi_{t} d x d t=\int_{0}^{t} \int_{-\infty}^{\infty}\left(p_{v}(\bar{v}, s)\left(\frac{\varphi^{2}}{2}\right)+Q_{1}\left(\frac{\varphi^{3}}{3}\right)\right) d x d t \\
=\left.\int_{-\infty}^{\infty}\left|p_{v}(\bar{v}, s)\right| \frac{\varphi^{2}}{2} d x\right|_{t=0} ^{t=t}+\left.O(1) N(t) \int_{-\infty}^{\infty} \phi^{2} d x\right|_{t=0} ^{t=t}
\end{gathered}
$$




$$
\begin{aligned}
& +\int_{0}^{t} \int_{-\infty}^{\infty}\left(p_{v v}(\bar{v}, s) \bar{v}_{t} \frac{\varphi^{2}}{2}+p_{v s}(\bar{v}, s) s_{t} \frac{\varphi^{2}}{2}\right) d x d t \\
& +\int_{0}^{t} \int_{-\infty}^{\infty}\left(Q_{1 v} \bar{v}_{t}+Q_{1 \varphi} \varphi_{t}+Q_{1 s} s_{t}\right) \frac{\varphi^{3}}{2} d x d t . \\
& =\left(\left|p_{v}^{-}\right|+O(1)\left(\delta_{0}+N(t)\right)\right)\left(\int_{-\infty}^{\infty} \frac{\varphi^{2}}{2}(t) d x-\int_{-\infty}^{\infty} \frac{\varphi^{2}(0)}{2} d x\right) \\
& +\left(p_{v v}^{-}+O(1)\left(\delta_{0}+N(t)\right)\right) \int_{0}^{t} \int_{-\infty}^{\infty} \bar{v}_{t} \frac{\varphi^{2}}{2} d x d t \\
& +\int_{0}^{t} \int_{-\infty}^{\infty}\left(p_{v s}(\bar{v}, s) s_{t} \frac{\varphi^{2}}{2}+Q_{1 \varphi} \varphi_{t} \frac{\varphi^{3}}{3}+Q_{1 s} s_{t} \frac{\varphi^{3}}{3}\right) d x d t .
\end{aligned}
$$

The above integrals which involve $s_{t}$ is estimated by (3.3) $)_{3}$ Lemma 2.1 , Sobolev and Young's inequalities as in previous estimates:

$$
\begin{aligned}
& \int_{0}^{t} \int_{-\infty}^{\infty}\left(p_{v s}(\bar{v}, s) s_{t} \frac{\varphi^{2}}{2}+Q_{1 s} s_{t} \frac{\varphi^{3}}{3}\right) d x d t \\
& \quad=O(1) N^{1 / 2}(t) \int_{0}^{t} \int_{-\infty}^{\infty}\left(s_{x}^{2}+\varphi_{x}^{2}+\psi_{x}^{2}\right) d x d t+O(1) N^{1 / 2}(t) \delta_{0}^{4 / 3},
\end{aligned}
$$

details are omitted. From (3.3) and the Sobolev inequality,

$$
\begin{aligned}
\left|\int_{0}^{t} \int_{-\infty}^{\infty} Q_{1 \varphi} \varphi_{t} \frac{\varphi^{3}}{2} d x d t\right| & \leqq \varepsilon_{1} \int_{0}^{t} \int_{-\infty}^{\infty} \psi_{x}^{2} d x d t+\frac{O(1)}{\varepsilon_{1}} \int_{0}^{t} \int_{-\infty}^{\infty}|\varphi|^{2}\|\varphi\|^{2}\left\|\varphi_{x}\right\|^{2} d x d t \\
& \leqq \varepsilon_{1} \int_{0}^{t} \int_{-\infty}^{\infty} \psi_{x}^{2} d x d t+\frac{O(1) N^{2}}{\varepsilon_{1}}(t) \int_{0}^{t} \int_{-\infty}^{\infty} \varphi_{x}^{2} d x d t \\
& =O(1) N(t) \int_{0}^{t} \int_{-\infty}^{\infty}\left(\psi_{x}^{2}+\varphi_{x}^{2}\right) d x d t
\end{aligned}
$$

if we set $\varepsilon_{1}=N(t)$. The above estimates yield

$$
\begin{aligned}
\int_{0}^{t} \int_{-\infty}^{\infty}[p(\bar{v}, s)-p(\bar{v}+\varphi, s)] \varphi_{t} d x d t \\
=\left(\left|p_{v}^{-}\right|+O(1)\left(\delta_{0}+N(t)\right)\right)\left(\int_{-\infty}^{\infty} \frac{\varphi^{2}(t)}{2} d x-\int_{-\infty}^{\infty} \frac{\varphi^{2}(0)}{2} d x\right) \\
\quad+\left(\left|p_{v v}^{-}\right|+O(1)\left(\delta_{0}+N(t)\right)\right) \int_{0}^{t} \int_{-\infty}^{\infty} v_{t} \frac{\varphi^{2}}{2} d x d t \\
\quad+O(1) N^{1 / 2}(t) \int_{0}^{t} \int_{-\infty}^{\infty}\left(s_{x}^{2}+\varphi_{x}^{2}+\psi_{x}^{2}\right) d x d t+O(1) N^{1 / 2}(t) \delta_{0}^{4 / 3}
\end{aligned}
$$

Similarly we have

$$
\int_{0}^{t} \int_{-\infty}^{\infty}(p(\bar{v}, 0)-p(\bar{v}, s)) \varphi_{t} d x d t=-\int_{0}^{t} \int_{-\infty}^{\infty}\left(p_{s}(\bar{v}, 0) s \varphi_{t}+Q_{2} s^{2} \varphi_{t}\right) d x d t
$$




$$
\begin{aligned}
= & -\left(p_{s}^{-}+\left.O(1)\left(\delta_{0}+N(t)\right) \int_{-\infty}^{\infty} s \varphi d x\right|_{t=0} ^{t=t}+\int_{0}^{t} \int_{-\infty}^{\infty}\left(p_{s}(\bar{v}, 0) s_{t} \varphi+p_{s v}(\bar{v}, 0) \bar{v}_{t} s \varphi\right.\right. \\
& \left.+Q_{2 v} \bar{v}_{t} s^{2} \varphi+Q_{2 s} s_{t} s^{2} \varphi\right) d x d t .
\end{aligned}
$$

The lowest order term on the right-hand side is estimated from (3.3) ${ }_{3}$ :

$$
\begin{aligned}
& \int_{0}^{t} \int_{-\infty}^{\infty}\left(p_{s}(\bar{v}, 0) s_{t} \varphi\right) d x d t=\frac{-\kappa p_{s}^{-}}{c_{v} v_{-}}\left(1+O(1)\left(N(t)+\delta_{0}\right)\right) \int_{0}^{t} \int_{-\infty}^{\infty} s_{x} \varphi_{x} d x d t \\
& \quad-\frac{(\gamma-1) \kappa}{v_{-}^{2}} p_{s}^{-}\left(1+O(1)\left(N(t)+\delta_{0}\right)\right) \int_{0}^{t} \int_{-\infty}^{\infty} \phi_{x}^{2} d x d t \\
& \quad+O(1)\left(\delta_{0}+N(t)\right) \int_{0}^{t} \int_{-\infty}^{\infty} \bar{v}_{t} \varphi^{2} d x d t \\
& \quad+O(1) N^{1 / 2}(t) \int_{0}^{t} \int_{-\infty}^{\infty}\left(s_{x}^{2}+\varphi_{x}^{2}+\psi_{x}^{2}\right) d x d t+O(1) N^{1 / 2}(t) \delta_{0}^{4 / 3} .
\end{aligned}
$$

Other terms are estimated similarly as above and we have

$$
\begin{aligned}
\int_{0}^{t} \int_{-\infty}^{\infty} & {[(p(\bar{v}, 0)-p(\bar{v}, s))] \varphi_{t} d x d t } \\
= & -\left(p_{s}^{-}+O(1)\left(\delta_{0}+N(t)\right)\left(\int_{-\infty}^{\infty} s(t) \varphi(t) d x-\int_{-\infty}^{\infty} s(0) \varphi(0) d x\right)\right. \\
& -\frac{\kappa p_{s}^{-}}{c_{v} v_{-}}\left(1+O(1)\left(N(t)+\delta_{0}\right)\right) \int_{0}^{t} \int_{-\infty}^{\infty} s_{x} \varphi_{x} d x d t \\
& +\frac{(\gamma-1) \kappa}{v_{-}^{2}} p_{s}^{-}\left(1+O(1)\left(N(t)+\delta_{0}\right)\right) \int_{0}^{t} \int_{-\infty}^{\infty} \varphi_{x}^{2} d x d t \\
& +O(1)\left(\delta_{0}+N(t)\right) \int_{0}^{t} \int_{-\infty}^{\infty} \bar{v}_{t} \varphi_{x}^{2} d x d t \\
& +O(1) N^{1 / 2}(t) \int_{0}^{t} \int_{-\infty}^{\infty}\left(s_{x}^{2}+\varphi_{x}^{2}+\psi_{x}^{2}\right) d x d t+O(1) N^{1 / 2}(t) \delta_{0}^{4 / 3}
\end{aligned}
$$

The lemma follows from (3.11) and the above estimates.

Q.E.D.

\section{Lemma 3.4.}

$$
\begin{aligned}
& \frac{\mu}{2 v_{-}} \int_{-\infty}^{\infty}\left|\varphi_{x}(t)\right|^{2} d x-\int_{-\infty}^{\infty} \varphi_{x}(t) \psi(t) d x+\left|p_{v}^{-}\right| \int_{0}^{t} \int_{-\infty}^{\infty} \varphi_{x}^{2} d x d t \\
& -p_{s}^{-} \int_{0}^{t} \int_{-\infty}^{\infty} s_{x} \varphi_{x} d x d t-\int_{0}^{t} \int_{-\infty}^{\infty} \psi_{x}^{2} d x d t \leqq O(1)\left(N^{2}(0)\right. \\
& +O(1)\left(\delta_{0}^{1 / 2}+N^{1 / 2}(t)\right)\left[\int_{-\infty}^{\infty}\left|\varphi_{x}(0)\right|^{2} d x+\int_{0}^{t} \int_{-\infty}^{\infty}\left(\psi_{x}^{2}+\varphi_{x}^{2}+s_{x}^{2}\right) d x d t\right] \\
& +O(1) N^{1 / 2}(t) \delta_{0}^{1 / 6}+O(1) \delta_{0}^{1 / 6} .
\end{aligned}
$$


Proof. Integrate $(3.3)_{2}$ times $\varphi_{x}$ and make use of $(3.3)_{1}$ to get

$$
\begin{aligned}
& \mu \int_{0}^{t} \int_{-\infty}^{\infty} \frac{\varphi_{x} \varphi_{x t}}{v+\varphi} d x d t-\int_{0}^{t} \int_{-\infty}^{\infty} p_{v}(\bar{v}+\varphi, s) \phi_{x}^{2} d x d t \\
& =\int_{0}^{t} \int_{-\infty}^{\infty}\left(\psi_{t} \varphi_{x}+\left(p_{v}(\bar{v}+\varphi, s)-p_{v}(\bar{v}, s)\right) \bar{v}_{x} \varphi_{x}+\left(p_{v}(\bar{v}, s)-p_{v}(\bar{v}, 0)\right) \bar{v}_{x} \varphi_{x}\right. \\
& \left.\quad+p_{s}(\bar{v}+\varphi, s) s_{x} \varphi_{x}-\mu\left(\frac{\bar{u}_{x}}{\bar{v}+\varphi}\right)_{x} \varphi_{x}+\mu \frac{\bar{v}_{x}+\varphi_{x}}{(\bar{v}+\varphi)^{2}} \varphi_{t} \varphi_{x}\right) d x d t
\end{aligned}
$$

We now estimate the terms in (3.13). Integration by parts (3.3), and Lemma 3.1 yield

$$
\begin{aligned}
\mu \int_{0}^{t} \int_{-\infty}^{\infty} \frac{\varphi_{x} \varphi_{x t}}{v+\varphi} d x d t=\left.\frac{\mu}{2} \int_{-\infty}^{\infty} \frac{\varphi_{x}^{2}}{v+\varphi} d x\right|_{t=0} ^{t=t}-\frac{\mu}{2} \int_{0}^{t} \int_{-\infty}^{\infty}\left(\frac{1}{\bar{v}+\varphi}\right) \varphi_{t}^{2} d x d t \\
=\frac{\mu}{2}\left(\frac{1}{v_{-}}+O(1)(\delta+N(t))\right) \int_{-\infty}^{\infty}\left(\varphi_{x}^{2}(t)-\varphi_{x}^{2}(0)\right) d x-\frac{\mu}{2} \int_{0}^{t} \int_{-\infty}^{\infty} O(1)\left(\bar{v}_{t}+\psi_{x}\right) \varphi_{x}^{2} d x d t \\
=\frac{\mu}{2}\left(\frac{1}{v_{-}}+O(1)(\delta+N(t))\right) \int_{-\infty}^{\infty}\left(\varphi_{x}^{2}(t)-\varphi_{x}^{2}(0)\right) d x+O(1) \frac{\mu}{2}(\delta+N(t)) \int_{0}^{t} \int_{-\infty}^{\infty} \varphi_{x}^{2} d x d t \\
\quad-\int_{0}^{t} \int_{-\infty}^{\infty} p_{v}(\bar{v}+\varphi, s) \varphi_{x}^{2} d x d t=\left(\left|p_{v}^{-}\right|+O(1)(\delta+N(t))\right) \int_{0}^{t} \int_{-\infty}^{\infty} \varphi_{x}^{2} d x d t .
\end{aligned}
$$

From integration by parts and $(3.3)_{1}$,

$$
\begin{aligned}
\int_{0}^{t} \int_{-\infty}^{\infty} \psi_{t} \varphi_{x} d x d t & =\left.\int_{-\infty}^{\infty} \psi \varphi_{x} d x\right|_{t=0} ^{t=t}-\int_{0}^{t} \int_{-\infty}^{\infty} \psi \psi_{x x} d x d t \\
& =\int_{-\infty}^{\infty}\left(\psi(t) \varphi_{x}(t)-\psi(0) \varphi_{x}(0)\right) d x+\int_{0}^{t} \int_{-\infty}^{\infty} \psi_{x}^{2} d x d t .
\end{aligned}
$$

From (ii) of Lemma 2.1 and Lemma 3.1,

$$
\begin{aligned}
& \int_{0}^{t} \int_{-\infty}^{\infty}\left(p_{v}(\bar{v}+\varphi, s)\right)-\left(p_{v}(\bar{v}, s)\right) \bar{v}_{x} \varphi_{x} d x d t=O(1) \int_{0}^{t} \int_{-\infty}^{\infty}\left|\varphi \bar{v}_{x} \varphi_{x}\right| d x d t \\
& \quad=O(1) \int_{0}^{t} \int_{-\infty}^{\infty}|\varphi|\left(\left|\bar{v}_{x}\right|^{2}+\varphi_{x}^{2}\right) d x d t \\
& =O(1) \int_{0}^{t}\|\varphi\|^{1 / 2}\left\|\varphi_{x}\right\|^{1 / 2}\left\|\bar{v}_{x}\right\|^{2} d t+O(1) N(t) \int_{0}^{t} \int_{-\infty}^{\infty} \varphi_{x}^{2} d x d t \\
& =O(1) \delta_{0} \int_{0}^{t}\left(\|\varphi\|^{1 / 2}\left\|\varphi_{x}\right\|^{2}+\|\varphi\|^{1 / 2}\left\|\bar{v}_{x}\right\|^{8 / 3}\right) d t+O(1) N(t) \int_{0}^{t} \int_{-\infty}^{\infty} \varphi_{x}^{2} d x d t \\
& \quad=O(1) N^{1 / 2}(t)\left(\int_{0}^{t}\left\|\varphi_{x}^{2}\right\| d t+\delta^{1 / 6}\right) .
\end{aligned}
$$

Similarly from Lemma 2.1 .

$$
\int_{0}^{t} \int_{-\infty}^{\infty}\left(p_{v}(\bar{v}, s)-p_{v}(\bar{v}, 0)\right) \bar{v}_{x} \varphi_{x} d x d t=O(1) \int_{0}^{t} \int_{-\infty}^{\infty}|s|\left|\bar{v}_{x} \varphi_{x}\right| d x d t
$$




$$
\begin{gathered}
=O(1) \int_{0}^{t} \int_{-\infty}^{\infty}|s|\left(\left|\bar{v}_{x}\right|^{2}+\left|\varphi_{x}\right|^{2}\right) d x d t \\
=O(1) N^{1 / 2}(t) \int_{0}^{t}\left(\left\|\varphi_{x}\right\|^{2}+\left\|s_{x}\right\|^{2}\right) d t+O(1) N^{1 / 2}(t) \delta^{1 / 6} \\
\int_{0}^{t} \int_{-\infty}^{\infty}-\mu\left(\frac{\bar{u}_{x}}{\bar{v}+\varphi}\right) \varphi_{x} \varphi_{x} d x d t=O(1) \int_{0}^{t} \int_{-\infty}^{\infty}\left(\left|\bar{u}_{x}\right|\left|\bar{v}_{x}\right|+\left|\bar{u}_{x}\right|\left|\varphi_{x}\right|+\left|\bar{u}_{x x}\right|\right)\left|\varphi_{x}\right| d x d t \\
=O(1) \delta_{0}^{1 / 2} \int_{0}^{t} \int_{-\infty}^{\infty} \varphi_{x}^{2} d x d t+O(1) \delta_{0}^{-1 / 2} \int_{0}^{t} \int_{-\infty}^{\infty}\left(\left|\bar{u}_{x}\right|^{4}+\left|\bar{u}_{x x}\right|^{2}\right) d x d t \\
=O(1) \delta_{0}^{1 / 2} \int_{0}^{t} \int_{-\infty}^{\infty} \varphi_{x}^{2} d x d t+O(1) \delta_{0}^{1 / 6}, \\
\int_{0}^{t} \int_{-\infty}^{\infty} \mu \frac{\left(\bar{v}_{x}+\varphi_{x}\right)}{(\bar{v}+\varphi)^{2}} \varphi_{t} \varphi_{x} d x d t=O(1) \int_{0}^{t} \int_{-\infty}^{\infty}\left|\bar{v}_{x}+\varphi_{x}\right| \psi_{x} \varphi_{x} d x d t \\
=O(1)\left(\delta_{0}+N(t)\right) \int_{0}^{t} \int_{-\infty}^{\infty}\left(\psi_{x}^{2}+\varphi_{x}^{2}\right) d x d t
\end{gathered}
$$

The lemma follows from (3.13) and the above estimates.

Q.E.D.

In the proof of the following key estimate we make use of the specific form of the constitutive relation (1.2).

Proposition 3.5. There exists a positive constant $\varepsilon$ such that if

$$
N(t)+\delta_{0} \leqq \varepsilon^{2}
$$

then

$$
\|(\varphi, \psi, s)(t)\|_{2}^{2}+\int_{0}^{t} \|\left(\varphi_{x}, \psi_{x}, s_{x} \|_{2}^{2}(t) d t \leqq C\left(N^{2}(0)+\delta_{0}^{1 / 6}\right)\right.
$$

for some $C$ independent of $t, N(0)$ and $\delta_{0}$.

Proof. The proof is based on Lemmas 3.2 3.4. From (1.2)

$$
p_{s}=p / c_{v}=\frac{(\gamma-1)}{R} p, \quad p_{v}=-\frac{\gamma p}{v}, \quad p_{v v}=\gamma(\gamma+1) \frac{p}{v^{2}}
$$

For simplicity we write $p=p_{-}, v \equiv v_{-}$etc., and set

$$
M \equiv \int_{-\infty}^{\infty}\left(\varphi(t)^{2}+\psi(t)^{2}+s(t)^{2}\right) d x+\int_{0}^{t} \int_{-\infty}^{\infty}\left(\varphi_{x}^{2}+\psi_{x}^{2}+s_{x}^{2}+\left|\bar{v}_{t}\right| \varphi^{2}\right) d x d t
$$

For $\varepsilon$ sufficiently small we have from Lemma $3.2 \sim 3.4$ and (3.16) that

$$
\begin{gathered}
\int_{-\infty}^{\infty} s^{2}(t) d x+\frac{2 \kappa(\gamma-1)}{R v} \int_{0}^{t} \int_{-\infty}^{\infty} s_{x}^{2} d x d t-\frac{2 \kappa(\gamma-1)}{v^{2}} \int_{0}^{t} \int_{-\infty}^{\infty} s_{x} \varphi_{x} d x d t \\
=O(1) \varepsilon M+O(1)\left(N^{2}(0)+\delta_{0}^{1 / 6}\right), \\
\int_{-\infty}^{\infty}\left(\psi^{2}(t)+\frac{\gamma p}{v} \varphi^{2}(t)-\frac{2(\gamma-1) p}{R} s(t) \varphi(t) d x\right.
\end{gathered}
$$




$$
\begin{aligned}
& +\int_{0}^{1} \int_{-\infty}^{\infty}\left(\frac{2 \mu}{v} \psi_{x}^{2}+\frac{2(\gamma-1)^{2} k p}{v^{2} R} \varphi_{x}^{2}+\frac{\gamma(\gamma+1) p}{2 v^{2}}\left|\bar{v}_{t}\right| \varphi^{2}-\frac{2(\gamma-1)^{2} k p}{R^{2} v} s_{x} \varphi_{x}\right) d x d t \\
= & O(1) N^{2}(0)+O(1) \varepsilon M+O(1) N^{1 / 2}(t) \delta_{0}^{1 / 6}+O(1) \varepsilon N^{2}(t) \\
& \int_{-\infty}^{\infty}\left(\frac{\mu}{2 v} \phi_{x}^{2}(t)-\varphi_{x}(t) \psi(t)\right) d x+\int_{0}^{t} \int_{-\infty}^{\infty}\left(\frac{\gamma p}{v} \varphi_{x}^{2}-\frac{(\gamma-1) p}{R} s_{x} \varphi_{x}-\psi_{x}^{2}\right) d x d t \\
& =O(1) N^{2}(0)+O(1) \varepsilon M+O(1) \delta_{0}^{1 / 6} .
\end{aligned}
$$

The terms on the left-hand side of $(3.18) \sim(3.20)$ which are not necessarily nonnegative are eliminated through linear combinations and the Cauchy-Schwartz inequality. Since the double integrals of $\psi_{x}^{2}$ appear only in (3.19) and (3.20) they can be eliminated by adding (3.19) times $v / 2 \mu$ and (3.20),

$$
\begin{aligned}
& \int_{-\infty}^{\infty}\left(\frac{v}{2 \mu} \psi^{2}(t)+\frac{\mu}{2 v} \varphi_{x}^{2}(t)-\varphi_{x}(t) \psi(t)+\frac{\gamma p}{2 \mu} \phi^{2}(t)-\frac{(\gamma-1) p v}{\mu R} s(t) \varphi(t) d x\right. \\
& \quad+\int_{0}^{t} \int_{-\infty}^{\infty}\left(\frac{(\gamma-1)^{2} \kappa p+\gamma \mu R p}{\mu v R} \varphi_{x}^{2}-\frac{(\gamma-1)^{2} \kappa p+(\gamma-1) \mu R p}{R^{2} \mu} s_{x} \varphi_{x}\right. \\
& \left.\quad+\frac{\gamma(\gamma+1) p}{6 \mu v}\left|\bar{v}_{t}\right| \varphi^{2}\right) d x d t \\
& =O(1) N^{2}(0)+O(1) \varepsilon M+O(1) N^{1 / 2}(t) \delta_{0}^{1 / 6}+O(1) \delta_{0}^{1 / 6} .
\end{aligned}
$$

Note that the first three terms above are positive definite:

$$
\int_{-\infty}^{\infty}\left(\frac{v}{2 \mu} \psi^{2}(t)-\phi_{x}(t) \psi(t)+\frac{\mu}{2 v} \varphi_{x}^{2}(t) d x\right) \geqq 0
$$

by the Cauchy-Schwartz inequality. Thus (3.21) yields

$$
\begin{gathered}
\int_{-\infty}^{\infty} \frac{\gamma}{2} \varphi^{2}(t)-\frac{(\gamma-1) v}{R} s(t) \varphi(t) d x+\int_{0}^{t} \int_{-\infty}^{\infty}\left(\frac{(\gamma-1)^{2} \kappa+\gamma \mu R}{v R} \varphi_{x}^{2}\right. \\
\left.-\frac{(\gamma-1)^{2} \kappa p+(\gamma-1) \mu R}{R^{2}} s_{x} \varphi_{x}+\frac{(\gamma+1)}{6 v}\left|\bar{v}_{t}\right| \varphi^{2}\right) d x d t \\
=O(1) N^{2}(0)+O(1) \varepsilon M+O(1) N^{1 / 2}(t) \delta_{0}^{1 / 6}+O(1) \delta_{0}^{1 / 6} .
\end{gathered}
$$

Add (3.22) to (3.18) times a variable $\alpha$,

$$
\begin{aligned}
\int_{-\infty}^{\infty}\left(\alpha s^{2}(t)+\frac{\gamma}{2} \varphi^{2}(t)-\frac{(\gamma-1) v}{R} s(t) \varphi(t)\right) d x & \\
& +\int_{0}^{t} \int_{-\infty}^{\infty} \frac{\gamma(\gamma+1)}{6 v}\left|\bar{v}_{t}\right| \varphi^{2} d x d t+\int_{0}^{t} \int_{-\infty}^{+\infty}\left(\frac{2 k(\gamma-1) \alpha}{R v} s_{x}^{2}+\frac{(\gamma-1)^{2} \kappa+\gamma \mu R}{v R} \varphi_{x}^{2}\right. \\
& \left.-\left(\frac{2 k(\gamma-1) \alpha}{v^{2}}+\frac{(\gamma-1)^{2} \kappa+(\gamma-1) \mu R}{R^{2}}\right) s_{x} \varphi_{x}\right) d x d t \\
= & O(1)\left(N^{2}(0)+\delta_{0}^{1 / 6}\right)+O(1) \varepsilon M+O(1) N^{1 / 2}(t) \delta_{0}^{1 / 6}
\end{aligned}
$$

The variable $\alpha$ is chosen so that the left-hand side of (3.23) is positive definite. For 
the single integral to be positive definite we need

$$
\alpha>\frac{(\gamma-1)^{2} v^{2}}{2 R^{2} \gamma} .
$$

For the double integral to be positive definite we need

$$
\Delta \equiv\left(\frac{2 \kappa(\gamma-1) \alpha}{v^{2}}+\frac{(\gamma-1)^{2} \kappa+(\gamma-1) \mu R}{R^{2}}\right)^{2}-\frac{8 \kappa(\gamma-1) \alpha\left[(\gamma-1)^{2} \kappa+\gamma \mu R\right]}{R^{2} v^{2}}<0
$$

for some $\alpha$ satisfying (3.24).

The expression $\Delta$ assumes minimum $\Delta_{\min }$ at $\alpha=\alpha_{\min }$ given by

$$
\begin{aligned}
\frac{4 \kappa(\gamma-1)}{v^{2}}\left(\frac{2 \kappa(\gamma-1)}{v^{2}} \alpha_{\min }+\frac{(\gamma-1)^{2} \kappa+(\gamma-1) \mu R}{R^{2}}\right) & \\
-\frac{8 \kappa(\gamma-1) \alpha\left[(\gamma-1)^{2} \kappa+\gamma \mu R\right]}{R^{2} v^{2}} & =0, \\
\alpha_{\min } & =\frac{(\gamma-1)^{2} \kappa+(1+\gamma) \mu R}{2 \kappa R^{2}(\gamma-1)} V^{2} .
\end{aligned}
$$

Clearly $\alpha=\alpha_{\min }$ satisfies (3.24) because $\gamma>1$. Direct calculations yield

$$
\begin{aligned}
\Delta & =\left(\frac{2 \kappa(\gamma-1) \alpha_{\min }}{v^{2}}+\frac{(\gamma-1)^{2} \kappa+(\gamma-1) \mu R}{R^{2}}\right)^{2}-\frac{8 \kappa(\gamma-1) \alpha_{\min }\left[(\gamma-1)^{2} \kappa+\gamma \mu R\right]}{R^{2} v^{2}} \\
& =\frac{\left.4(\gamma-1)^{2} \kappa+\gamma \mu R\right)^{2}}{R^{4}}-\frac{\left.\left.4(\gamma-1)^{2} \kappa+(\gamma-1)^{2} \mu R\right)^{2}(\gamma-1)^{2} \kappa+\gamma \mu R\right)}{R^{4}} \\
& =\frac{\left.-4(\gamma-1)^{2} \mu \kappa R\right)^{2}-4 \gamma \mu^{2} R^{2}}{R^{4}}<0 .
\end{aligned}
$$

This shows that the integrals in (3.23) are positive definite and so:

$$
\begin{aligned}
& \int_{-\infty}^{\infty}\left(s^{2}(t)+\varphi^{2}(t)\right) d x+\int_{0}^{t} \int_{-\infty}^{\infty}\left|\bar{v}_{t}\right| \varphi^{2} d x d t+\int_{0}^{t} \int_{-\infty}^{\infty}\left(s_{x}^{2}+\psi_{x}^{2}\right) d x d t \\
& =O(1)\left(N^{2}(0)+\delta_{0}^{1 / 6}\right)+O(1) \varepsilon M+O(1) N^{1 / 2}(t) \delta_{0}^{1 / 6} .
\end{aligned}
$$

This and (3.19), (3.20) finally yield

$$
\begin{aligned}
& \int_{-\infty}^{\infty}\left(s^{2}(t)+\varphi^{2}(t)+\psi^{2}(t)+\varphi_{x}^{2}(t)\right) d x+\int_{0}^{t} \int_{-\infty}^{\infty}\left(\left|\bar{v}_{t}\right| \varphi^{2}+s_{x}^{2}+\varphi_{x}^{2}+\varphi_{x}^{2}\right) d x d t \\
& =O(1)\left(N(0)+\delta_{0}^{1 / 6}\right)+O(1) \varepsilon M+O(1) N^{1 / 2}(t) \delta_{0}^{1 / 6} .
\end{aligned}
$$

In particular, we have from (3.17) that for $\varepsilon$ small,

$$
\begin{aligned}
& \int_{-\infty}^{\infty}\left(s^{2}(t)+\varphi^{2}(t)+\psi^{2}(t)+\varphi_{x}^{2}(t)\right) d x+\int_{0}^{t} \int_{-\infty}^{\infty}\left(\left|\bar{v}_{t}\right| \varphi^{2}+s_{x}^{2}+\varphi_{x}^{2}+\varphi_{x}^{2}\right) d x d t \\
& =O(1)\left(N^{2}(0)+\delta_{0}^{1 / 6}\right)+O(1) N^{1 / 2}(t) \delta^{1 / 6} .
\end{aligned}
$$

This completes the estimate of the lower order terms on the left-hand side of (3.15). 
The higher order terms are estimated in a similar way using the energy method applied to the derivatives of (3.3). Since the argument is exactly the same, we omit the details. Thus we have

$$
\|(\varphi, \psi, s)(t)\|_{2}^{2}+\int_{0}^{t}\left\|\varphi_{x}, \psi_{x}, s_{x}\right\|_{2}^{2}(t) d t=O(1)\left(N^{2}(0)+\delta_{0}^{1 / 6}\right)+O(1) N^{1 / 2}(t) \delta^{1 / 6} .
$$

In particular we have from (3.6) that

$$
N^{2}(t)=O(1)\left(N^{2}(0)+\delta_{0}^{1 / 6}\right)+O(1) N^{1 / 2}(t) \delta_{0}^{1 / 6},
$$

which implies, for $N^{2}(0)$ and $\delta_{0}$ small,

$$
N^{2}(t)=O(1)\left(N^{2}(0)+\delta_{0}^{1 / 6}\right) .
$$

This and (3.25) finally yield (3.15).

\section{Existence and Asymptotic Behavior}

With proposition 3.5 the proof of our main theorem becomes routine. First, the hypothesis (3.14) is implied by (3.15) under the assumption that $N(0)$ and $\delta_{0}$ are small. This observation and the usual local existence theorem for the hyperbolic parabolic system (1.1), or (3.3) yield the global existence theorem.

The asymptotic behavior is the consequence of the Sobolev inequality, Proposition 3.5 and the equicontinuity of $\left\|s_{x}(t)\right\|,\left\|\varphi_{x}(t)\right\|$ and $\left\|\psi_{x}(t)\right\|$ in $t$, which is contained in the estimate of these terms. Since we did not carry out the detail of the estimate, we now illustrate the equicontinuity of $\left\|\psi_{x}(t)\right\|$ by integrating $\psi_{x}(3.3)_{2 x}$ over $-\infty<x<\infty, t_{1} \leqq t \leqq t$ :

$$
\begin{aligned}
\frac{1}{2} \int_{-\infty}^{\infty}\left(\psi_{x}^{2}\left(t_{2}\right)-\psi_{x}^{2}\left(t_{1}\right)\right) d x= & \int_{t_{1}}^{t_{2}} \int_{-\infty}^{\infty}-(p(\varphi+\bar{v}, s)-p(\bar{v}, 0))_{x x} \psi_{x} d x d t \\
& +\mu \int_{t_{1}}^{t_{2}} \int_{-\infty}^{\infty}\left(\frac{\bar{u}_{x}+\psi_{x}}{\bar{v}+\varphi}\right)_{x} \psi_{x x} d x d t .
\end{aligned}
$$

By carrying out the above derivatives on the right-hand side we have

$$
\begin{aligned}
\frac{1}{2} \int_{-\infty}^{\infty}\left(\psi_{x}^{2}\left(t_{2}\right)-\psi_{x}^{2}\left(t_{1}\right)\right) d x \\
=O(1) \int_{i_{1}}^{t_{2}} \int_{-\infty}^{\infty}\left(\left|\varphi_{x x}\right|+\left|s_{x x}\right|+\left|\bar{v}_{x}\right|^{2}+\left|\bar{v}_{x x}\right|+\left|\psi_{x}\right|^{2}+\left|s_{x}\right|^{2}\right) d x d t \\
\quad-\mu \int_{t_{1}}^{t_{2}} \int_{-\infty}^{\infty} \frac{\left|\psi_{x x}\right|^{2}}{\bar{v}+\varphi} d x d t+O(1) \int_{t_{1}}^{t_{2}} \int_{-\infty}^{\infty}\left(\left|\bar{u}_{x x}\right|+\left|\bar{u}_{x}\right|^{2}+\left|\varphi_{x}\right|^{2}+\left|\psi_{x}\right|^{2}\right)\left|\psi_{x x}\right| d x d t
\end{aligned}
$$

which yields, by Lemma 2.1 and the Cauchy-Schwartz inequality

$$
\begin{aligned}
\int_{-\infty}^{\infty} \varphi_{x}^{2}\left(t_{2}\right) d x= & \int_{-\infty}^{\infty} \varphi_{x}^{2}\left(t_{1}\right) d x+O(1) \int_{i_{1}}^{t_{2}} \int_{-\infty}^{\infty}\left(\left|\varphi_{x}\right|^{2}+\left|s_{x}\right|^{2}+\left|\psi_{x}\right|^{2}+\left|\psi_{x x}\right|^{2}\right. \\
& \left.+\left|\varphi_{x x}\right|^{2}+\left|s_{x x}\right|^{2}\right) d x d t+O(1) \delta_{0}\left(1+t_{1}\right)^{-1}, \quad t_{2}>t_{1} \geqq 0 .
\end{aligned}
$$


Similar estimates also hold for $\left\|\varphi_{x}(t)\right\|$ and $\left\|s_{x}(t)\right\|$ :

$$
\begin{aligned}
\left\|\left(\varphi_{x}, \psi_{x}, s_{x}\right)\left(t_{2}\right)\right\|= & \left\|\left(\varphi_{x}, \psi_{x}, s_{x}\right)\left(t_{1}\right)\right\|+O(1) \int_{t_{1}}^{t_{2}}\left\|\varphi_{x}, \psi_{x}, s_{x}\right\|^{2}(t) d t \\
& +O(1) \delta_{0}\left(1+t_{1}\right)^{-1}, \quad t_{2}>t_{1} \geqq 0 .
\end{aligned}
$$

By integrating with respect to the variable $t_{1}$ we have

$$
\left\|\left(\varphi_{x}, \psi_{x}, s_{x}\right)(t)\right\|=O(1) \int_{t}^{t}\left\|\left(\varphi_{x}, \psi_{x}, s_{x}\right)(t)\right\| d t+O(1) \delta_{0}(1+t)^{-1}, \quad t \geqq 1
$$

From Proposition 3.5, (3.15), for arbitrary given $\varepsilon$, there exists $t_{0}>0$ such that

$$
\int_{i_{0}}^{\infty}\left\|\varphi_{x}, \psi_{x}, s_{x}\right\|_{1}^{2} d t \leqq \varepsilon
$$

For any $t>t_{0}+1$, we have from the Sobolev inequality, (4.1) and (3.15) that

$$
\begin{aligned}
\sup _{-\infty<x<\infty}|(\varphi, \psi, s)(x, t)| & \leqq\|(\varphi, \psi, s)(t)\| \cdot\left\|\left(\varphi_{x}, \psi_{x}, s_{x}\right)(t)\right\| \\
& \leqq O(1)\left(N(0)+\delta_{0}^{12}\right)\left\|\left(\varphi_{x}, \psi_{x}, s_{x}\right)(t)\right\| \\
& \leqq O(1)\left(N(0)+\delta_{0}^{12}\right)\left(\varepsilon+\delta_{0}(1+t)^{-1}\right) .
\end{aligned}
$$

Since $\varepsilon$ is arbitrary, we finally conclude

$$
\lim _{t \rightarrow \infty} \sup _{-\infty<\chi<\infty}|(\varphi, \psi, s)(x, t)|=0 .
$$

This proves our main theorem.

\section{References}

1. Courant, R., Friedrichs, K.O.: Supersonic flows and shock waves. Berlin, Heidelberg, New York: Springer

2. Liu, T..P.: Linear and nonlinear large-time behaviors of solutions of hyperbolic conservation laws. Commun. Pure Appl. Math. 30 (1977)

3. Liu, T.-P.: Shock waves for compressible Navier-Stokes equations are stable. Commun. Pure Appl. Math. 39, 565-594 (1986)

4. Liu T.-P.: Hyperbolic conservation laws with relaxation. Commun. Math. Phys. 108, 153-175 (1986)

5. Matzumura, A., Nishihara, K.: Asymptotics toward the rarefaction waves of the solutions of a one-dimensional model system for compressible viscous gas. Jpn J. Appl. Math. 3, 1-13 (1986)

6. Xin, Z.P.: Asymptotic stability of rarefaction waves for $2 \times 2$ viscous hyperbolic conservation laws. J. Differ. Eqs. 72 (1988)

Communicated by A. Jaffe

Received March 8, 1988 